\title{
General Expressions for the Circular Constant $\pi$
}

\author{
Chunyang Ma \\ School of Mechanical and Electrical Engineering, Nanchang University, Nanchang, China \\ Email: cymaphy@163.com,cyma@ncu.edu.cn
}

How to cite this paper: Ma, C.Y. (2021) General Expressions for the Circular Constant $\pi$. Applied Mathematics, 12, 18-23. https://doi.org/10.4236/am.2021.121002

Received: December 1, 2020

Accepted: January 18, 2021

Published: January 21, 2021

Copyright $\odot 2021$ by author(s) and Scientific Research Publishing Inc. This work is licensed under the Creative Commons Attribution International License (CC BY 4.0).

http://creativecommons.org/licenses/by/4.0/

\begin{abstract}
From ancient times to the present, mathematicians have put forward many series expressions of the circular constant. Because of the importance of the circular constant to mathematical physics, the research on circular constant has never stopped. In this paper, the general function expression of the circular constant was given by studying the transient heat conduction equation. From the physical aspect of the derivation process of the circular constant expression, we can conclude that there is an infinite number of different series exist that can be used to express $\pi$.
\end{abstract}

\section{Keywords}

Circular Constant, Infinite Series, Pi

\section{Introduction}

The calculation of circular constant has a long history [1] [2]. Many mathematicians have proposed elegant calculation expressions for the circular constant [3] [4] [5]. This research has no purpose to solve any specific problems. It is hoped that it will bring new enlightenment and enrich its connotation to the research on circular constant. Inspired by the problem of transient heat conduction, based on the theory of Fourier series, I proposed a general expression for expressing the circular constant. It was discovered that there can be infinite different series expressions for circular constant. I hope this general expression can bring new inspiration to the rapid calculation of the value of circular constant and its possible potential applications in mathematical physics.

\section{Series Expressions of the Circular Constant}

A general expression of the circular constant $\pi$ was derived. The start point is to study the unsteady heat transfer problem for different initial conditions, whereas with the same initial average temperature. Without losing generality, the 
one-dimensional transient heat conduction equation can be written as [6]

$$
\frac{\partial T}{\partial t}=a \frac{\partial^{2} T}{\partial x^{2}}
$$

With initial conditions: 1) the sample with uniform distribution of temperature, I; 2) the sample with linear distribution of temperature, II; 3) using the solution 1) at time $t_{c}$ as the initial temperature distribution, III.

$$
\begin{gathered}
\text { I: } t=0,0 \leq x \leq L, T(x)=T_{\text {ave }} \\
\text { II: } t=0,0 \leq x \leq L, T(0)=0, T(L)=T_{L}, T(x)=\frac{T_{L}}{L} x ;
\end{gathered}
$$

III: $t=0,0 \leq x \leq L, T(x, 0)=\frac{4 T}{\pi} \sum_{n} \exp \left[-a\left(\frac{2 n+1}{2 L} \pi\right)^{2} t_{c}\right] \frac{1}{2 n+1} \sin \left(\frac{2 n+1}{2 L} \pi x\right)(4)$

The average temperature of the three cases is equally, therefore, we have

$$
\begin{gathered}
T_{\text {ave }}=\frac{4 T}{\pi^{2}} \sum_{n} \exp \left[-a\left(\frac{2 n+1}{2 L} \pi\right)^{2} t_{c}\right] \frac{2}{(2 n+1)^{2}} \\
T_{L}=2 T_{\text {ave }}
\end{gathered}
$$

and the boundary conditions are one side at temperature $0^{\circ} \mathrm{C}$, and the other side is heat insulation. Mathematically

$$
\begin{aligned}
& x=0, T(0, t)=0 \\
& x=L,\left.\frac{\mathrm{d} T}{\mathrm{~d} x}\right|_{x=L}=0
\end{aligned}
$$

The separation of variables method can be applied to solve the transient heat conduction equation, by assuming

$$
T(x, t)=g(x) f(t)
$$

Therefore, we can obtain solutions of a discrete series

$$
\begin{aligned}
& f_{n}(t)=\exp \left[-a\left(\frac{2 n+1}{2 L} \pi\right)^{2} t\right] \\
& g_{n}(x)=2 H_{n} \sin \left(\frac{2 n+1}{2 L} \pi x\right)
\end{aligned}
$$

where $n=0,1, \cdots, N$, and $H_{n}$ is the coefficient for $\mathrm{n}^{\text {th }}$ series. The value of $H_{n}$ can be obtained by the initial condition, for initial condition I, II, and III, respectively

$$
\begin{gathered}
\sum_{n} 2 H_{n}^{\mathrm{I}} \sin \left(\frac{2 n+1}{2 L} \pi x\right)=T_{\text {ave }} \\
\sum_{n} 2 H_{n}^{\mathrm{II}} \sin \left(\frac{2 n+1}{2 L} \pi x\right)=\frac{T_{L}}{L} x \\
\sum_{n} 2 H_{n}^{\mathrm{III}} \sin \left(\frac{2 n+1}{2 L} \pi x\right)=\frac{4 T}{\pi} \sum_{n} \exp \left[-a\left(\frac{2 n+1}{2 L} \pi\right)^{2} t_{c}\right] \frac{1}{2 n+1} \sin \left(\frac{2 n+1}{2 L} \pi x\right)
\end{gathered}
$$


The $H_{n}^{\alpha}$ can be obtained by the Fourier's orthogonal series [7], which $\alpha=$ I, II, III . By multiply $\sin \left(\frac{2 m+1}{2 L} \pi x\right)$ for both sides, and integral within the interval $[0, L]$

$$
\begin{gathered}
H_{n}^{\mathrm{I}}=\frac{T_{\text {ave }}}{\pi} \frac{2}{2 n+1} \\
H_{n}^{\mathrm{II}}=(-1)^{n} \frac{T_{L}}{\pi^{2}}\left(\frac{2}{2 n+1}\right)^{2} \\
H_{n}^{\mathrm{III}}=\frac{2 T}{\pi} \mu_{n}
\end{gathered}
$$

which the Fourier's integral $\int_{0}^{L} \sin \left(\frac{2 n+1}{2 L} \pi x\right) \sin \left(\frac{2 n+1}{2 L} \pi x\right) \mathrm{d} x=\frac{L}{2}$ is used, and the expression of $\mu_{n}$ is defined as $\mu_{n}:=\exp \left[-a\left(\frac{2 n+1}{2 L} \pi\right)^{2} t_{c}\right] \frac{1}{2 n+1}$, which the symbol := represents the identity definition. Therefore, the solutions of the three cases can be solved, respectively

$$
\begin{gathered}
T^{\mathrm{I}}(x, t)=\frac{4 T_{\text {ave }}}{\pi} \sum_{n} \exp \left[-a\left(\frac{2 n+1}{2 L} \pi\right)^{2} t\right] \frac{1}{2 n+1} \sin \left(\frac{2 n+1}{2 L} \pi x\right) \\
T^{\mathrm{II}}(x, t)=\frac{8 T_{L}}{\pi^{2}} \sum_{n} \exp \left[-a\left(\frac{2 n+1}{2 L} \pi\right)^{2} t\right](-1)^{n}\left(\frac{1}{2 n+1}\right)^{2} \sin \left(\frac{2 n+1}{2 L} \pi x\right) \\
T^{\mathrm{III}}(x, t)=\frac{4 T}{\pi} \sum_{n} \mu_{n} \exp \left[-a\left(\frac{2 n+1}{2 L} \pi\right)^{2} t\right] \sin \left(\frac{2 n+1}{2 L} \pi x\right)
\end{gathered}
$$

Averaging the temperature with respect to space coordinate $x$, we can obtain

$$
\begin{gathered}
T^{\mathrm{I}}(t)=\frac{1}{L} \int_{0}^{L} T^{\mathrm{I}}(x, t) \mathrm{d} x=\frac{4 T_{\text {ave }}}{\pi^{2}} \sum_{n} \exp \left[-a\left(\frac{2 n+1}{2 L} \pi\right)^{2} t\right] \frac{2}{(2 n+1)^{2}} \\
T^{\mathrm{II}}(t)=\frac{1}{L} \int_{0}^{L} T^{\mathrm{II}}(x, t) \mathrm{d} x=\frac{16 T_{L}}{\pi^{3}} \sum_{n} \exp \left[-a\left(\frac{2 n+1}{2 L} \pi\right)^{2} t\right](-1)^{n}\left(\frac{1}{2 n+1}\right)^{3} \\
T^{\mathrm{III}}(t)=\frac{1}{L} \int_{0}^{L} T^{\mathrm{III}}(x, t) \mathrm{d} x=\frac{4 T}{\pi^{2}} \sum_{n} \exp \left[-a\left(\frac{2 n+1}{2 L} \pi\right)^{2}\left(t+t_{c}\right)\right] \frac{2}{(2 n+1)^{2}}
\end{gathered}
$$

which the relation $\mu_{n}:=\exp \left[-a\left(\frac{2 n+1}{2 L} \pi\right)^{2} t_{c}\right] \frac{1}{2 n+1}$ is used. The average temperature should be equal for the three cases, when $t=0$, thus,

$$
\begin{gathered}
T^{\mathrm{I}}(0)=\frac{4 T_{\text {ave }}}{\pi^{2}} \sum_{n} \frac{2}{(2 n+1)^{2}} \\
T^{\mathrm{II}}(0)=\frac{16 T_{L}}{\pi^{3}} \sum_{n}(-1)^{n}\left(\frac{1}{2 n+1}\right)^{3}
\end{gathered}
$$




$$
T^{\mathrm{III}}(0)=\frac{4 T}{\pi^{2}} \sum_{n} \exp \left[-a\left(\frac{2 n+1}{2 L} \pi\right)^{2} t_{c}\right] \frac{2}{(2 n+1)^{2}}:=T_{\text {ave }}
$$

The Equations (24) to (26) can lead to

$$
\begin{gathered}
\pi=\frac{\sum_{n}(-1)^{n}\left(\frac{2}{2 n+1}\right)^{3}}{\sum_{n} \frac{2}{(2 n+1)^{2}}} \\
\pi^{2}=\sum_{n} \frac{8}{(2 n+1)^{2}} \\
\pi^{3}=32 \sum_{n}(-1)^{n}\left(\frac{1}{2 n+1}\right)^{3}
\end{gathered}
$$

We can see that mathematics and physics are inseparable, and the connection between them is so wonderful. In this way, the series expression of $\pi, \pi^{2}$, and $\pi^{3}$ are obtained corresponding to three different initial conditions used here. The functional form of the initial conditions is infinite, and the possible expressions of $\pi^{n}$ can even be obtained from this, hence, we can imagine that there is infinite number of different series exist can be used to express $\pi$. For a general initial temperature distribution, written as following

$$
t=0,0 \leq x \leq L, T(x, 0)=h(x)
$$

Similarly, we can obtain the solution

$$
\begin{aligned}
T(x, t)= & \frac{2}{\pi} \sum_{n} \exp \left[-a\left(\frac{2 n+1}{2 L} \pi\right)^{2} t\right] \frac{2}{2 n+1} \sin \left(\frac{2 n+1}{2 L} \pi x\right) \\
& \cdot\left[h_{+}(0)+\int_{0}^{L} \cos \left(\frac{2 n+1}{2 L} \pi x\right) \mathrm{d} h(x)\right]
\end{aligned}
$$

which the coefficients of the Fourier's orthogonal series expressed as

$$
H_{n}=\frac{2}{2 n+1} \frac{1}{\pi}\left[h_{+}(0)+\int_{0}^{L} \cos \left(\frac{2 n+1}{2 L} \pi x\right) \mathrm{d} h(x)\right]
$$

Averaging the temperature with respect to space coordinate $x$ over the length $L$, therefore,

$$
\begin{aligned}
T(t)= & \frac{4}{\pi^{2}} \sum_{n} \exp \left[-a\left(\frac{2 n+1}{2 L} \pi\right)^{2} t\right] \frac{2}{(2 n+1)^{2}} \\
& \cdot\left[h_{+}(0)+\int_{0}^{L} \cos \left(\frac{2 n+1}{2 L} \pi x\right) \mathrm{d} h(x)\right]
\end{aligned}
$$

Making $h(x)$ satisfies the normalization condition $\frac{1}{T_{a v e} L} \int_{0}^{L} h(x) \mathrm{d} x=1$, and $t=0$, corresponding to the initial average temperature. Therefore, we can obtain

$$
\pi^{2}=\frac{2 \sum_{n}\left(\frac{2}{2 n+1}\right)^{2}\left[h_{+}(0)+\int_{0}^{L} \cos \left(\frac{2 n+1}{2 L} \pi x\right) d h(x)\right]}{\frac{1}{L} \int_{0}^{L} h(x) \mathrm{d} x}
$$


Here, the $h(x)$ is an arbitrary (temperature) distribution function, which periodic with period $L$. We can deduce that the constant $\pi$ can be expressed by infinitely different kinds of series, which due to the function $h(x)$ have infinitely choices. Further, can we conjecture that any mathematical and physical constants can be expressed by infinitely different kinds of series? Combining Equation (33) with Equation (24) and let $t=0$, we obtain

$$
\sum_{n} \frac{2}{(2 n+1)^{2}}\left[h_{+}(0)+\int_{0}^{L} \cos \left(\frac{2 n+1}{2 L} \pi x\right) \mathrm{d} h(x)\right]=\frac{1}{L} \int_{0}^{L} h(x) \mathrm{d} x \sum_{n} \frac{2}{(2 n+1)^{2}}
$$

where the $\pi$ is disappeared in the expression, which states that Equation (35) is valid for arbitrary function $h(x)$. Similarly, combining Equation (33) with Equation (25) and let $t=0$, therefore, we obtain an expression of $\pi$

$$
\pi=\frac{\frac{1}{L} \int_{0}^{L} h(x) \mathrm{d} x \sum_{n}(-1)^{n}\left(\frac{2}{2 n+1}\right)^{3}}{\sum_{n} \frac{2}{(2 n+1)^{2}}\left[h_{+}(0)+\int_{0}^{L} \cos \left(\frac{2 n+1}{2 L} \pi x\right) \mathrm{d} h(x)\right]}
$$

where $h(x)$ is an arbitrary function, which satisfies the normalization condition $\frac{1}{T_{\text {ave }} L} \int_{0}^{L} h(x) \mathrm{d} x=1$.

\section{Conclusion}

A general mathematical expression of the universally circular constant $\pi$ was derived. The circular constant can be expressed by infinitely different kinds of series for the infinitely different choices of the initial distribution function. From this, can we conjecture that any mathematical and physical constants can be expressed by infinitely different kinds of series?

\section{Acknowledgements}

This study was supported by the funding from Nanchang University. Thank you for my wife and family, this paper wouldn't have been possible without your selfless care and unconditional love for our family.

\section{Conflicts of Interest}

The author declares no conflicts of interest regarding the publication of this paper.

\section{References}

[1] Agarwal, R.P., Agarwal, H. and Sen, S.K. (2013) Birth, Growth and Computation of pi to Ten Trillion Digits. Advances in Difference Equations, 2013, Article Number: 100. https://doi.org/10.1186/1687-1847-2013-100

[2] Finch, S.R. (2003) Mathematical Constants. Cambridge University Press, Cambridge. https://doi.org/10.1017/CBO9780511550447

[3] Borwein, J.M. and Borwein, P.B. (1993) Class Number Three Ramanujan Type Se- 
ries for 1/ $\pi$. Journal of Computational and Applied Mathematics, 46, 281-290. https://doi.org/10.1016/0377-0427(93)90302-R

[4] Lange, L.J. (1999) An Elegant Continued Fraction for pi. American Mathematical Monthly, 106, 456-458. https://doi.org/10.1080/00029890.1999.12005070

[5] Sun, Z.W. (2020) New Series for Powers of pi and Related Congruences. Electronic Research Archive, 28, 1273-1342. https://doi.org/10.3934/era.2020070

[6] Incropera, F.P. and Dewitt, D.P. (2006) Fundamentals of Heat and Mass Transfer. John Wiley \& Sons, New York.

[7] Powers, D.L. (2006) Boundary Value Problems and Partial Differential Equations. Academic Press, Oxford. 